\title{
ON SPACES FILLED UP BY CONTINUOUS COLLECTIONS OF ATRIODIC CONTINUOUS CURVES
}

\author{
R. D. ANDERSON AND MARY-ELIZABETH HAMSTROM
}

Throughout this paper, $G$ will denote a nondegenerate continuous collection of atriodic continuous curves (i.e. arcs or simple closed curves) filling up a compact metric continuum $M$. As is well known, we may regard $G$ itself as a compact metric continuum, with the elements of the collection $G$ as the points of the space $G$ and with $G$, as a space, the image of $M$ under an open continuous mapping whose inverse sets are the elements of the collection $G$.

The principal results of this paper are the following theorems.

Theorem I. No closed, totally disconnected point set separates $M .^{1}$

Theorem II. If each element of $G$ is an arc, then no closed, totally disconnected point set separates any connected open subset of $M$.

Theorem III. If $G$ is a two-dimensional Cantor manifold, ${ }^{2}$ then $M$ is not separated by any rational curve. ${ }^{3}$

As an immediate corollary of Theorem I, we have

Theorem IV. ${ }^{4}$ At no point of $M$ is the dimension of $M$ less than 2.

A well known result cited in [2, Theorem VI7, page 91] states, in effect, that if $\operatorname{dim} M-\operatorname{dim} G=k>0$, then at least one element of $G$ has dimension not less than $k$. From Theorem IV, this result, and the fact that every atriodic continuous curve is one-dimensional, we obtain

Theorem V. If $G$ is a one-dimensional continuum, then $M$ is twodimensional at each of its points.

Presented to the Society, December 27, 1954; received by the editors December 7, 1954.

1 The theorem of [1] shows emphatically that Theorem I cannot be strengthened to the extent of deleting the condition that the continuous curves of $G$ be atriodic.

${ }^{2}$ An $n$-dimensional Cantor manifold is a compact metric $n$-dimensional space which is not separated by any $(n-2)$-dimensional subspace.

${ }^{3} \mathrm{~A}$ rational curve is a compact metric continuum $K$ such that each point of $K$ is contained in arbitrarily small neighborhoods relative to $K$ whose boundaries are countable. It is to be noted that a rational curve is not necessarily locally connected.

${ }^{4}$ Eldon Dyer has recently obtained some general and interesting theorems about the dimension of $G$ if $M$ is $n$-dimensional and $G$ is a continuous collection of arcs (or dendrons). 
We shall give a proof of Theorem I in some detail. The proofs of Theorems II and III are similar to that of Theorem I. We shall simply indicate the arguments for these.

A simple chain in $M$ is a finite collection $x_{1}, x_{2}, \cdots, x_{n}$ of open sets such that $x_{i} \cdot x_{j}$ exists if and only if $|i-j| \leqq 1$. The sets $x_{1}, x_{2}, \cdots, x_{n}$ are called the links of the chain. We note that if $s$ is an open interval of the arc $t$ which lies in $M$, there is an open subset $U$ of $M$ such that $U \cdot t=s$ and $\bar{U} \cdot \bar{t}=\bar{s}$. If $t$ is an arc and $\epsilon$ is any positive number, then there exists a simple chain $C$ covering $t$ each of whose links is of diameter less than $\epsilon$ and intersects $t$ in a connected set.

A special case of a result of $\mathrm{J}$. H. Roberts [3, Theorem 2] states that if $E$ is a continuous collection of arcs filling up a compact metric space, there is a subcollection $E^{\prime}$ of $E$ such that $E^{\prime}$ is dense in $E$ and $E$ is equicontinuous ${ }^{5}$ at each element of $E^{\prime}$. An argument suggested by that outlined in [3] yields a similar result in the case where $E$ is a continuous collection of atriodic continuous curves. We do not give this argument in detail but henceforth let $G^{\prime}$ be a subcollection of $G$ at each element of which $G$ is equicontinuous.

Proof of Theorem I. Suppose, contrary to the statement of Theorem I that some closed, totally disconnected point set $T$ separates $M$ into sets $D_{1}$ and $D_{2}$. Then, since $M$ is connected and $G$ is continuous, some element $g_{1}$ of $G$ intersects each of $D_{1}$ and $D_{2}$ and, since $G^{\prime}$ is dense in $G$, some element $g$ of $G^{\prime}$ intersects each of $D_{1}$ and $D_{2}$. Let $h$, with endpoints $a_{1}$ and $a_{2}$ in $D_{1}$ and $D_{2}$ respectively, be an arc in $g$. For each $i, i=1,2$, let $A_{i}$ be an open set containing $a_{i}$ with $\bar{A}_{i}$ contained in $D_{i}$. Let $C: c_{1}, \cdots, c_{5}$ be a simple chain covering $h$ such that (1) $c_{1}-c_{1} \cdot c_{2}$ contains $A_{1}$, (2) $c_{5}-c_{4} \cdot c_{5}$ contains $A_{2}$, (3) $\bar{c}_{1}+\bar{c}_{2}$ is a subset of $D_{1},(4) \bar{c}_{4}+\bar{c}_{5}$ is a subset of $D_{2},(5)$ for $i=1, \cdots, 5$, $\bar{c}_{i} \cdot h$ is connected, and (6) for $i=2,3,4, c_{i}$ and $g-h$ are mutually exclusive. Clearly such a chain exists. Let $U$ containing $g$ be an open subcollection of $G$ such that each element $u$ of $U$ contains an arc $u_{x}$ with endpoints in $A_{1}$ and $A_{2}$ respectively such that (1) $u_{x}$ is covered by $C$, (2) $u-u_{x}$ does not intersect $\bar{c}_{3}$, and (3) $u_{x}$ does not contain two disjoint arcs each intersecting $A_{1}$ and $c_{3}, A_{2}$ and $c_{3}$, or $c_{2}$ and $c_{4}$. From the equicontinuity of $G$ at $g$ it follows that such a set $U$ exists.

Let $\bar{G}$ containing $g$ be a nondegenerate closed connected subcollection of $U$ and let $\widetilde{H}$ containing $h$ be a collection of arcs in one-to-one correspondence with $\widetilde{G}$ such that each element of $\widetilde{H}$ is contained in

5 The collection $G$ as above is said to be equicontinuous at the element $g$ of $G$ provided that for any $\epsilon>0$ and any point $p$ of $g$, there exists a $\delta>0$ such that if $x$ and $y$ are points of the same element $g^{\prime}$ of $G$ and are each within $\delta$ of $p$, then there is an arc in $g^{\prime}$ containing $x+y$ and of diameter less than $\epsilon$. 
the corresponding element of $\tilde{G}$, is covered by $C$, and has its endpoints in $A_{1}$ and $A_{2}$ respectively. Let $\widetilde{H}$ be topologized so as to be homeomorphic with $\tilde{G}$ under the correspondence above.

Let $g^{\prime}$ be an element of $\widetilde{G}$ distinct from $g$ and let $h^{\prime}$ be the corresponding element of $\tilde{H}$. Let $W$ be an open set in $c_{3}-\left(c_{2}+c_{4}\right) \cdot c_{3}$ such that $\bar{W}-W$ does not intersect $T, W$ contains $T \cdot h$, and $W$ does not intersect $h^{\prime}$. Let $Z_{1}$ and $Z_{2}$ be the subsets of $\bar{W}-W$ in $D_{1}$ and $D_{2}$ respectively. Each of $Z_{1}$ and $Z_{2}$ is closed.

For each element $k$ of $\widetilde{H}$, let $N(k)$ be the collection of those components of $k \cdot W$ having limit points in each of $Z_{1}$ and $Z_{2}$. Let $n(k)$ be the number $(\bmod 2)$ of elements in $N(k)$. Let $\widetilde{H}_{0}$ be the collection of all elements $k$ of $\widetilde{H}$ for which $n(k)=0$. The collection $\widetilde{H}_{0}$ contains $h^{\prime}$ and $\widetilde{H}-\widetilde{H}_{0}$ contains $h$. We wish to show that each of $\widetilde{H}_{0}$ and $\widetilde{H}-\widetilde{H}_{0}$ is open and hence that $\widetilde{G}$ is not connected-a contradiction.

Let $\tilde{h}$ be any element of $\widetilde{H}$. There exists a simple chain $C(\tilde{h})$ covering $\tilde{h}$ such that (1) each link of $C(\tilde{h})$ is a subset of a link of $C$ and intersects $\tilde{h}$ and (2) no link of $C(\tilde{h})$ intersects (a) each of $D_{1}$ and $D_{2}$ but not $T$, (b) each of $Z_{1}$ and $Z_{2}$, or (c) each of $\bar{W}$ and $T-T \cdot W$.

Let $X(\tilde{h})$ be the collection of links of $C(\tilde{h})$ intersecting $\bar{W}$. Let $Y(\tilde{h})$ be the collection of all maximal simple chains whose links are elements of $X(\tilde{h})$. If $k$ is any element of $\tilde{H}$ containing a subarc $k^{\prime}$ with endpoints in $A_{1}$ and $A_{2}$ and with $k^{\prime}$ covered by $C(\tilde{h})$, then $n(k)=n(\tilde{h})$. This follows from the fact that each element of $N(k)$ is covered by exactly one element of $Y(\tilde{h})$ and the number of elements of $N(k)$ in an element $y$ of $Y(\tilde{h})$ is 1 or $0(\bmod 2)$ according as the end links of $y$ do or do not intersect different sets of the two sets $Z_{1}$ and $Z_{2}$. Hence $\widetilde{H}_{0}$ and $\widetilde{H}-\widetilde{H}_{0}$ are each open and Theorem I is proved.

IndicATION OF PROOF OF TheOREM II. Suppose, contrary to Theorem II that there exists a connected open set $D$ in $M$ separated by a closed and totally disconnected set $T$. Let $\left(D_{1}, D_{2}\right)$ be a separation of $D$ by $T$. Then some element of $G$ must contain an arc in $D$ with endpoints in $D_{1}$ and $D_{2}$ respectively. Since $G$ is a collection of arcs, some element of $G^{\prime}$ must also contain an arc in $D$ with endpoints in $D_{1}$ and $D_{2}$ respectively and the argument is essentially reduced to that for Theorem I. We note that if the elements of $G$ are not restricted to being arcs, then there exist simple examples without such an element of $G^{\prime}$ existing and with an open set separated by a point.

InDICATION OF PROOF OF ThEOREM III. Suppose $G$ is a two-dimensional Cantor manifold and $M$ is separated by a rational curve $J$ into the two mutually separated sets $D_{1}$ and $D_{2}$. Clearly some element of $G$ must intersect each of $D_{1}$ and $D_{2}$ for otherwise the set of those elements of $G$ lying completely in $J$ must exist and be closed 
and in $G$ must separate $G$. But by Theorem I this set is 0-dimensional, a contradiction. Hence some element of $G$ intersects each of $D_{1}$ and $D_{2}$, and thus some element of $G^{\prime}$ contains an arc $h$ with endpoints in $D_{1}$ and $D_{2}$ respectively. But by an argument similar to that used in the proof of Theorem I we can exhibit an open subcollection $U$ of $G$ with $U$ containing $g$ and with $\bar{U}-U$ countable, a contradiction.

\section{REFERENCES}

1. R. D. Anderson, Continuous collections of continuous curves, Duke Math. J. vol. 21 (1954) pp. 363-368.

2. Witold Hurewicz and Henry Wallman, Dimension theory, Princeton University Press, 1948.

3. J. H. Roberts, Collections filling a plane, Duke Math. J. vol. 2 (1936) pp. 10-19.

The University of Pennsylvania and

Goucher College

\section{A NOTE ON BASIC SETS OF HOMOGENEOUS HARMONIC POLYNOMIALS}

E. P. MILES, JR. AND E. WILliamS

For any set of non-negative integers $\left(b_{j}\right)$ such that $b_{1} \leqq 1$ and $\sum_{j=1}^{k} b_{j}=n$, let

$$
H_{b_{1} \ldots b_{k}}^{n}=\sum(-1)^{\left[a_{1} / 2\right]} \frac{n !}{\prod_{j=1}^{k} a_{j} !} \cdot \frac{\left[\frac{a_{1}}{2}\right] !}{\prod_{j=2}^{k}\left(\frac{b_{j}-a_{j}}{2}\right) !} \prod_{j=1}^{k} x_{j}^{a_{j}}
$$

where the summation is extended over all $\left(a_{j}\right)$ such that,

(a) $a_{j} \equiv b_{j} \bmod 2, j=1,2, \cdots, k$,

(b) $\sum_{j=1}^{\boldsymbol{k}} a_{j}=n$,

(c) $a_{j} \leqq b_{j}, j=2,3, \cdots, k$.

The polynomials (1) were shown by the authors to form a basic set of homogeneous harmonic polynomials in $k$ variables [1]. ${ }^{1}$

It is easily seen that the following differential recursion formulas hold for these polynomials:

Presented to the Society, November 26, 1954 under the title $A$ basic set of homogeneous polynomials in $k$ variables. II; received by the editors November 29, 1954.

${ }^{1}$ Numbers in brackets refer to bibliography at the end of the paper. 